\title{
Tables as Semi-structured Knowledge for Question Answering
}

\author{
Sujay Kumar Jauhar \\ Carnegie Mellon University \\ Pittsburgh, PA, USA \\ sjauharecs.cmu.edu
}

\author{
Peter D. Turney \\ Allen Institute for Artificial Intelligence \\ Seattle, WA, USA \\ peterteallenai.org
}

\author{
Eduard Hovy \\ Carnegie Mellon University \\ Pittsburgh, PA, USA \\ hovy@cs. cmu . edu
}

\begin{abstract}
Question answering requires access to a knowledge base to check facts and reason about information. Knowledge in the form of natural language text is easy to acquire, but difficult for automated reasoning. Highly-structured knowledge bases can facilitate reasoning, but are difficult to acquire. In this paper we explore tables as a semi-structured formalism that provides a balanced compromise to this tradeoff. We first use the structure of tables to guide the construction of a dataset of over 9000 multiple-choice questions with rich alignment annotations, easily and efficiently via crowd-sourcing. We then use this annotated data to train a semistructured feature-driven model for question answering that uses tables as a knowledge base. In benchmark evaluations, we significantly outperform both a strong unstructured retrieval baseline and a highlystructured Markov Logic Network model.
\end{abstract}

\section{Introduction}

Question answering (QA) has emerged as a practical research problem for pushing the boundaries of artificial intelligence (AI). Dedicated projects and open challenges to the research community include examples such as Facebook AI Research's challenge problems for AI-complete QA (Weston et al., 2015) and the Allen Institute for AI's (AI2) Aristo project (Clark, 2015) along with its recently completed Kaggle competition ${ }^{1}$. The reason for this emergence is the diversity of core language and reasoning problems that a complex, integrated

\footnotetext{
${ }^{1}$ https: / / www.kaggle.com/c/ the-allen-ai-science-challenge
}

task like QA exposes: information extraction (Srihari and Li, 1999), semantic modelling (Shen and Lapata, 2007; Narayanan and Harabagiu, 2004), logic and reasoning (Moldovan et al., 2003), and inference (Lin and Pantel, 2001).

Complex tasks such as QA require some form of knowledge base to store facts about the world and reason over them. By knowledge base, we mean any form of knowledge: structured (e.g., tables, ontologies, rules) or unstructured (e.g., natural language text). For QA, knowledge has been harvested and used in a number of different modes and formalisms: large-scale extracted and curated knowledge bases (Fader et al., 2014), structured models such as Markov Logic Networks (Khot et al., 2015), and simple text corpora in information retrieval approaches (Tellex et al., 2003).

There is, however, a fundamental trade-off in the structure and regularity of a formalism and its ability to be curated, modelled or reasoned with easily. For example, simple text corpora contain no structure, and are therefore hard to reason with in a principled manner. Nevertheless, they are easily and abundantly available. In contrast, Markov Logic Networks come with a wealth of theoretical knowledge connected with their usage in principled inference. However, they are difficult to induce automatically from text or to build manually.

In this paper we explore tables as semistructured knowledge for multiple-choice question (MCQ) answering. Specifically, we focus on tables that represent general knowledge facts, with cells that contain free-form text (Secton 3 details the nature and semantics of these tables). The structural properties of tables, along with their free-form text content represents a semi-structured balanced compromise in the trade-off between degree of structure and ubiquity. We present two main contributions, with tables and their structural properties playing a crucial role in both. First, 
we crowd-source a collection of over $9000 \mathrm{MCQs}$ with alignment annotations to table elements, using tables as guidelines in efficient data harvesting. Second, we develop a feature-driven model that uses these MCQs to perform QA, while factchecking and reasoning over tables.

Others have used tables in the context of QA. Question bank creation for tables has been investigated (Pasupat and Liang, 2015), but without structural guidelines or the alignment information that we propose. Similarly, tables have been used in QA reasoning (Yin et al., 2015b; Neelakantan et al., 2015; Sun et al., 2016) but have not explicitly attempted to encode all the semantics of table structure (see Section 3.1). To the best of our knowledge, no previous work uses tables for both creation and reasoning in a connected framework.

We evaluate our model on MCQ answering for three benchmark datasets. Our results consistently and significantly outperform a strong retrieval baseline as well as a Markov Logic network model (Khot et al., 2015). We thus show the benefits of semi-structured data and models over unstructured or highly-structured counterparts. We also validate our curated MCQ dataset and its annotations as an effective tool for training QA models. Finally, we find that our model learns generalizations that permit inference when exact answers may not even be contained in the knowledge base.

\section{Related Work}

Our work with tables, semi-structured knowledge bases and QA relates to several parallel lines of research. In terms of dataset creation via crowdsourcing, Aydin et al. (2014) harvest MCQs via a gamified app, although their work does not involve tables. Pasupat and Liang (2015) use tables from Wikipedia to construct a set of QA pairs. However their annotation setup does not impose structural constraints from tables, and does not collect finegrained alignment to table elements.

On the inference side Pasupat and Liang (2015) also reason over tables to answer questions. Unlike our approach, they do not require alignments to table cells. However, they assume knowledge of the table that contains the answer, a priori - which we do not. Yin et al. (2015b) and Neelakantan et al. (2015) also use tables in the context of QA, but deal with synthetically generated query data. Sun et al. (2016) perform cell search over web tables via relational chains, but are more generally inter- ested in web queries. Clark et al. (2016) combine different levels of knowledge for QA, including an integer-linear program for searching over table cells. None of these other efforts leverage tables for generation of data.

Our research more generally pertains to natural language interfaces for databases. Answering questions in this context refers to executing queries over relational databases (Cafarella et al., 2008; Pimplikar and Sarawagi, 2012). Yin et al. (2015a) consider databases where information is stored in n-tuples, which are essentially tables. Also, investigation of the relational structure of tables is connected with research on database schema analysis and induction (Venetis et al., 2011; Syed et al., 2010). Finally, unstructured text and structured formats links to work on open information extraction (Etzioni et al., 2008) and knowledge base population (Ji and Grishman, 2011).

\section{Tables as Semi-structured Knowledge Representation}

Tables can be found on the web containing a wide range of heterogenous data. To focus and facilitate our work on QA we select a collection of tables that were specifically designed for the task. Specifically we use AI2's Aristo Tablestore ${ }^{2}$. However, it should be noted that the contributions of this paper are not tied to specific tables, as we provide a general methodology that could equally be applied to a different set of tables. The structural properties of this class of tables is further described in Section 3.1.

The Aristo Tablestore consists of 65 handcrafted tables organized by topic. Some of the topics are bounded, containing only a fixed number of facts, such as the possible phase changes of matter (see Table 1). Other topics are unbounded, containing a very large or even infinite number of facts, such as the kind of energy used in performing an action (the corresponding tables can only contain a sample subset of these facts). A total of 3851 facts (one fact per row) are present in the manually constructed tables. An individual table has between 2 and 5 content columns.

The target domain for these tables is two 4th grade science exam datasets. The majority of the tables were constructed to contain topics and facts

\footnotetext{
2http://allenai.org/content/data/ AristoTablestore-Nov2015Snapshot.zip
} 


\begin{tabular}{ccccccc}
\hline Phase Change & & Initial State & & Final State & Form of Energy Transfer \\
\hline Melting & causes a & solid & to change into a & liquid & by & adding heat \\
Vaporization & causes a & liquid & to change into a & gas & by & adding heat \\
Condensation & causes a & gas & to change into a & liquid & by & removing heat \\
Sublimation & causes a & solid & to change into a & gas & by & adding heat \\
\hline
\end{tabular}

Table 1: Part of a table concerning phase changes in matter. Rows are facts. Columns without header text provide filler text, so that each row forms a sentence. In columns with header text, the header describes the type of entry in the column; the header is a hypernym of the text in the body below.

from the publicly available Regents dataset ${ }^{3}$. The rest were targeted at an unreleased dataset called Monarch. In both cases only the training partition of each dataset was used to formulate and handcraft tables. However, for unbounded topics, additional facts were added to each table, using science education text books and websites.

\subsection{Table Semantics and Relations}

Part of a table from the Aristo Tablestore is given as an example in Table 1. The format is semistructured: the rows of the table (with the exception of the header) are a list of sentences, but with well-defined recurring filler patterns. Together with the header, these patterns divide the rows into meaningful columns. This semi-structured data format is flexible. Since facts are presented as sentences, the tables can act as a text corpus for information retrieval. At the same time the structure can be used - as we do - to focus on specific nuggets of information. The flexibility of these tables allows us to compare our table-based system to an information retrieval baseline.

Such tables have some interesting structural semantics, which we will leverage throughout the paper. A row in a table corresponds to a fact $^{4}$. The cells in a row correspond to concepts, entities, or processes that participate in this fact. A content column ${ }^{5}$ corresponds to a group of concepts, entities, or processes that are the same type. The header cell of the column is an abstract description of the type. We may view the head as a hypernym and the cells in the column below as co-hyponyms of the head. The header row defines a generalization of which the rows in the table are specific instances.

This structure is directly relevant to multiplechoice QA. Facts (rows) form the basis for creat-

\footnotetext{
${ }^{3}$ http://allenai.org/content/data/ Regents.zip

${ }^{4}$ Also predicates, or more generally frames with typed arguments.

${ }^{5}$ Different from filler columns, which only contain a recurring pattern, and no information in their header cells.
}

ing or answering questions, while instances of a type (columns) act as the choices of an MCQ. We use these observations both for crowd-sourcing MCQ creation as well as for designing features to answer MCQs with tables.

\section{Crowd-sourcing Multiple-choice Questions from Tables}

We use Amazon's Mechanical Turk (MTurk) service to generate MCQs by imposing constraints derived from the structure of the tables. These constraints help annotators create questions with scaffolding information, and lead to consistent quality in the generated output. An additional benefit of this format is the alignment information, linking cells in the tables to the MCQs generated by the Turkers. The alignment information is generated as a by-product of making the MCQs.

We present Turkers with a table such as the one in Figure 1. Given this table, we choose a target cell to be the correct answer for a new MCQ; for example, the red cell in Figure 1. First, Turkers create a question by using information from the rest of the row containing the target (i.e., the blue cells in Figure 1), such that the target is its correct answer. Then they select the cells in the row that they used to construct the question. Following this, they construct four succinct choices for the question, one of which is the correct answer and the other three are distractors. Distractors are formed from other cells in the column containing the target (i.e. yellow cells in Figure 1). If there are insufficient unique cells in the column Turkers create their own. Annotators can rephrase and shuffle the contents of cells as required.

In addition to an MCQ, we obtain alignment information with no extra effort from annotators. We know which table, row, and column contains the answer, and thus we know which header cells might be relevant to the question. We also know the cells of a row that were used to construct a question. 


\begin{tabular}{||c|c|c|c|c|c|c|}
\hline PHASE CHANGE & & INITIAL PHASE & & FINAL PHASE & & HEAT TRANSFER \\
\hline \hline Melting & causes $\mathrm{a}$ & solid & to change into $\mathrm{a}$ & liquid & by & adding heat \\
\hline \hline Vaporization & causes $\mathrm{a}$ & liquid & to change into $\mathrm{a}$ & gas & by & adding heat \\
\hline Sublimation & causes $\mathrm{a}$ & solid & to change into $\mathrm{a}$ & gas & by & adding heat \\
\hline \hline Freezing & causes $\mathrm{a}$ & liquid & to change into $\mathrm{a}$ & solid & by & removing heat \\
\hline \hline Deposition & causes $\mathrm{a}$ & gas & to change into $\mathrm{a}$ & solid & by & removing heat \\
\hline \hline Condensation & causes a & gas & to change into a & liquid & by & removing heat \\
\hline
\end{tabular}

Figure 1: Example table from MTurk annotation task illustrating constraints. We ask Turkers to construct questions from blue cells, such that the red cell is the correct answer, and yellow cells form distractors.

\begin{tabular}{llll}
\hline Task & Avg. Time (s) & \$/hour & \% Reject \\
\hline Rewrite & 345 & 2.61 & 48 \\
Paraphrase & 662 & 1.36 & 49 \\
Add choice & 291 & 2.47 & 24 \\
Write new & 187 & $\mathbf{5 . 7 8}$ & 38 \\
TabMCQ & $\mathbf{7 2}$ & 5.00 & $\mathbf{2}$ \\
\hline
\end{tabular}

Table 2: Comparison of different ways of generating MCQs with MTurk.

\begin{tabular}{l} 
What is the orbital event with \\
the longest day and the shortest night? \\
\hline A) Summer solstice \\
B) Winter solstice \\
C) Spring equinox \\
D) Fall equinox \\
\hline Steel is a/an ----- of electricity \\
A) Separator \\
B) Isolator \\
C) Insulator \\
D) Conductor
\end{tabular}

Table 3: Examples of MCQs generated by MTurk. Correct answer choices are in bold.

\subsection{The TabMCQ Dataset}

We created a HIT (the MTurk acronym for Human Intelligence Task) for every non-filler cell (see Section 3) from each one of the 65 manually constructed tables of the Aristo Tablestore. We paid annotators 10 cents per MCQ, and asked for 1 annotation per HIT for most tables. For an initial set of four tables which we used in a pilot study, we asked for three annotations per HIT $^{6}$. We required Turkers to have a HIT approval rating of $95 \%$ or higher, with a minimum of at least 500 HITs approved. We restricted the demographics of our workers to the US.

Table 2 compares our method with other studies conducted at AI2 to generate MCQs. These methods attempt to generate new MCQs from existing

\footnotetext{
${ }^{6}$ The goal was to obtain diversity in the MCQs created for a target cell. The results were not sufficiently conclusive to warrant a threefold increase in the cost of creation.
}

ones, or write them from scratch, but do not involve tables in any way. Our annotation procedure leads to faster data creation, with consistent output quality that resulted in the lowest percentage of rejected HITs. Manual inspection of the generated output also revealed that questions are of consistently good quality. They are good enough for training machine learning models and many are good enough as evaluation data for QA. A sample of generated MCQs is presented in Table 3.

We implemented some simple checks to evaluate the data before approving HITs. These included things like checking whether an MCQ has at least three choices and whether choices are repeated. We had to further prune our data to discard some MCQs due to corrupted data or badly constructed MCQs. A total of 159 MCQs were lost through the cleanup. In the end our complete data consists of 9092 MCQs, which is - to the best of our knowledge - orders of magnitude larger than any existing collection of science exam style MCQs available for research. These MCQs also come with alignment information to tables, rows, columns and cells. The dataset, bundled together with the Aristo Tablestore, can be freely downloaded ${ }^{7}$.

\section{Solving MCQs with Table Cell Search}

Consider the MCQ "What is the process by which water is changed from a liquid to a gas?" with choices "melting, sublimation, vaporization, condensation", and the table given in Figure 1. Finding the correct answer amounts to finding a cell in the table that is most relevant to a candidate QA pair. In other words, a relevant cell should confirm the assertion made by a particular QA pair.

By applying the reasoning used to create MCQs

\footnotetext{
${ }^{7}$ http://ai2-website.s3.amazonaws.com/ data/TabMCQ_v_1.0.zip
} 
(see Section 4) in the inverse direction, finding these relevant cells becomes the task of finding an intersection between rows and columns of interest. Consider the table in Figure 1: assuming we have some way of aligning a question to a row (blue cells) and choices to a column (yellow cells), then the relevant cell is at the intersection of the two (the red cell). This alignment is precisely what we get as a by-product of the annotation task we setup in Section 4 to harvest MCQs.

We can thus featurize connections between MCQs and elements of tables and use the alignment data to train a model over the features. This is outlined in the next section, describing our Feature Rich Table Embedding Solver (FRETS).

\subsection{Model and Training Objective}

Let $\mathcal{Q}=\left\{q_{1}, \ldots, q_{N}\right\}$ denote a set of MCQs, and $\mathcal{A}_{n}=\left\{a_{n}^{1}, \ldots, a_{n}^{k}\right\}$ be the set of candidate answer choices for a given question $q_{n}$. Let the set of tables be defined as $\mathcal{T}=\left\{T_{1}, \ldots, T_{M}\right\}$. Given a table $T_{m}$, let $t_{m}^{i j}$ be the cell in that table corresponding to the $i$ th row and $j$ th column.

We define a log-linear model that scores every cell $t_{m}^{i j}$ of every table in our collection according to a set of discrete weighted features, for a given QA pair. We have the following:

$$
\begin{aligned}
& \log p\left(t_{m}^{i j} \mid q_{n}, a_{n}^{k} ; \mathcal{A}_{n}, \mathcal{T}\right)= \\
& \quad \sum_{d} \lambda_{d} f_{d}\left(q_{n}, a_{n}^{k}, t_{m}^{i j} ; \mathcal{A}_{n}, \mathcal{T}\right)-\log Z
\end{aligned}
$$

Here $\lambda_{d}$ are weights and $f_{d}\left(q_{n}, a_{n}^{k}, t_{m}^{i j} ; \mathcal{A}_{n}, \mathcal{T}\right)$ are features. These features should ideally leverage both structure and content of tables to assign high scores to relevant cells, while assigning low scores to irrelevant cells. $Z$ is the partition function, defined as follows:

$$
\begin{aligned}
& Z= \\
& \sum_{m, i, j} \exp \left(\sum_{d} \lambda_{d} f_{d}\left(q_{n}, a_{n}^{k}, t_{m}^{i j} ; \mathcal{A}_{n}, \mathcal{T}\right)\right)
\end{aligned}
$$

$Z$ normalizes the scores associated with every cell over all the cells in all the tables to yield a probability distribution. During inference the partition term $\log Z$ can be ignored, making scoring cells of every table for a given QA pair efficient.

These scores translate to a solution for an MCQ. Every QA pair produces a hypothetical fact, and as noted in Section 3.1, the row of a table is in essence a fact. Relevant cells (if they exist) should confirm the hypothetical fact asserted by a given QA pair. During inference, we assign the score of the highest scoring row (or the most likely fact) to a hypothetical QA pair. Then the correct solution to the MCQ is simply the answer choice associated with the QA pair that was assigned the highest score. Mathematically, this is expressed as follows:

$$
\begin{aligned}
a_{n}^{*}=\underset{a_{n}^{k}}{\arg \max } \max _{m, i} & \\
& \sum_{j} \sum_{d} \lambda_{d} f_{d}\left(q_{n}, a_{n}^{k}, t_{m}^{i j} ; \mathcal{A}_{n}, \mathcal{T}\right)
\end{aligned}
$$

\subsubsection{Training}

Since FRETS is a log-linear model, training involves optimizing a set of weights $\lambda_{d}$. As training data, we use alignment information between MCQs and table elements (see Section 4.1). The predictor value that we try to maximize with our model is an alignment score that is closest to the true alignments in the training data. True alignments to table cells for a given QA pair are essentially indicator values but we convert them to numerical scores as follows ${ }^{8}$. For a correct QA hypothesis we assign a score of 1.0 to cells whose row and column and both aligned to the MCQ (i.e. cells that exactly answer the question), 0.5 to cells whose row but not column is aligned in some way to the question (i.e. cells that were used to construct the question), and 0.0 otherwise. For an incorrect QA hypothesis we assign a score of 0.1 to random cells from tables that contain no alignments to the QA (so all except one), with a probability of $1 \%$, while all other cells are scored 0.0. The intuition behind this scoring scheme is to guide the model to pick relevant cells for correct answers, while encouraging it to pick faulty evidence with low scores for incorrect answers.

Given these scores assigned to all cells of all tables for all QA pairs in the training set, suitably normalized to a probability distribution over tables for a given QA pair, we can then proceed to train our model. We use cross-entropy, which minimizes the following loss:

\footnotetext{
${ }^{8}$ On training data, we experimented with a few different scoring heuristics and found that these ones worked well.
} 


\begin{tabular}{|c|c|c|c|c|c|}
\hline Level & Feature & Description & Intuition & S-Var & Cmpct \\
\hline \multirow{2}{*}{ Table } & Table score & Ratio of words in $\mathbf{t}$ to $\mathbf{q}+\mathbf{a}$ & Topical consistency & $\diamond$ & \\
\hline & $\dagger$ TF-IDF table score & Same but TF-IDF weights & Topical consistency & $\diamond$ & \\
\hline \multirow{3}{*}{ Row } & Row-question score & Ratio of words in $\mathbf{r}$ to $\mathbf{q}$ & Question align & $\diamond$ & \\
\hline & Row-question w/o focus score & Ratio of words in $\mathbf{r}$ to $\mathbf{q}-\left(\mathbf{a}_{\mathbf{f}}+\mathbf{q}_{\mathbf{f}}\right)$ & Question align & $\diamond$ & \\
\hline & Header-question score & Ratio of words in $\mathbf{h}$ to $\mathbf{q}$ & Prototype align & $\diamond$ & \\
\hline \multirow{3}{*}{ Column } & Column overlap & Ratio of elements in $\mathbf{c}$ and $\mathbf{A}$ & Choices align & $\diamond$ & \\
\hline & Header answer-type match & Ratio of words in $\mathbf{c}_{\mathbf{h}}$ to $\mathbf{a}_{\mathbf{f}}$ & Choices hypernym align & $\diamond$ & \\
\hline & Header question-type match & Ratio of words in $\mathbf{c}_{\mathbf{h}}$ to $\mathbf{q}_{\mathbf{f}}$ & Question hypernym align & $\diamond$ & \\
\hline \multirow{3}{*}{ Cell } & †Cell salience & Salience of $\mathbf{s}$ to $\mathbf{q}+\mathbf{a}$ & QA hypothesis assert & $\diamond$ & \\
\hline & $\dagger$ Cell answer-type entailment & Entailment score between $\mathbf{s}$ and $\mathbf{a}_{\mathbf{f}}$ & Hypernym-hyponym align & & \\
\hline & Cell answer-type similarity & Avg. vector sim between $\mathbf{s}$ and $\mathbf{a}_{\mathbf{f}}$ & Hypernym-hyponym sim. & & \\
\hline
\end{tabular}

Table 4: Summary of features. For a question (q) and answer (a) we compute scores for elements of tables: whole tables $(\mathbf{t})$, rows $(\mathbf{r})$, header rows $(\mathbf{h})$, columns $(\mathbf{c})$, column headers $\left(\mathbf{c}_{\mathbf{h}}\right)$ and cells $(\mathbf{s})$. Answer-focus $\left(\mathbf{a}_{\mathbf{f}}\right)$ and question-focus $\left(\mathbf{q}_{\mathbf{f}}\right)$ terms added where appropriate. Features marked $\diamond$ denote soft-matching variants, marked with while those marked with a $\dagger$ are described in further detail in Section 5.2. Finally, features denote those that received high weights during training with all features, and were subsequently selected to form a compact FRETS model.

$$
\begin{aligned}
L(\vec{\lambda})= & \sum_{\substack{q_{n} \\
a_{n}^{k} \in \mathcal{A}_{n}}} \sum_{m, i, j} p\left(t_{m}^{* i j} \mid q_{n}, a_{n}^{k} ; \mathcal{T}\right) . \\
& \log p\left(t_{m}^{i j} \mid q_{n}, a_{n}^{k} ; \mathcal{A}_{n}, \mathcal{T}\right)
\end{aligned}
$$

Here $p\left(t_{m}^{* i j} \mid q_{n}, a_{n}^{k} ; \mathcal{T}\right)$ is the normalized probability of the true alignment scores.

While this is an indirect way to train our model to pick the best answer, in our pilot experiments it worked better than direct maximum likelihood or ranking with hinge loss, achieving a training accuracy of almost $85 \%$. Our experimental results on the test suite, presented in the next section, also support the empirical effectiveness of this approach.

\subsection{Features}

The features we use are summarized in Table 4 . These features compute statistics between question-answer pairs and different structural components of tables. While the features are weighted and summed for each cell individually, they can capture more global properties such as scores associated with tables, rows or columns in which the specific cell is contained. Features are divided into four broad categories based on the level of granularity at which they operate. In what follows we give some details of Table 4 that require further elaboration.

\subsubsection{Soft matching}

Many of the features that we implement are based on string overlap between bags of words. However, since the tables are defined statically in terms

of a fixed vocabulary (which may not necessarily match words contained in an MCQ), these overlap features will often fail. We therefore soften the constraint imposed by hard word overlap by a more forgiving soft variant. More specifically we introduce a word-embedding based soft matching overlap variant for every feature in the table marked with $\diamond$. The soft variant targets high recall while the hard variant aims at providing high precision. We thus effectively have almost twice the number of features listed.

Mathematically, let a hard overlap feature define a score $\left|S_{1} \cap S_{2}\right| /\left|S_{1}\right|$ between two bags of words $S_{1}$ and $S_{2}$. We can define the denominator $S_{1}$ here, without loss of generality. Then, a corresponding word-embedding soft overlap feature is given by this formula:

$$
\frac{1}{\left|S_{1}\right|} \sum_{w_{i} \in S_{1}} \max _{w_{j} \in S_{2}} \operatorname{sim}\left(\vec{w}_{i}, \vec{w}_{j}\right)
$$

Intuitively, rather than matching a word to its exact string match in another set, we instead match it to its most similar word, discounted by the score of that similarity.

\subsubsection{Question parsing}

We parse questions to find the desired answertype and, in rarer cases, question-type words. For example, in the question "What form of energy is required to convert water from a liquid to a gas?", the type of the answer we are expecting is a "form of energy". Generally, this answer-type corresponds to a hypernym of the answer choices, and can help find relevant information in the table, specifically related to columns. 
By carefully studying the kinds of question patterns in our data, we implemented a rule-based parser that finds answer-types from queries. This parser uses a set of hand-coded regular expressions over phrasal chunks. The parser is designed to have high accuracy, so that we only produce an output for answer-types in high confidence situations. In addition to producing answer-types, in some rarer cases we also detect hypernyms for parts of the questions. We call this set of words question-type words. Together, the question-type and answer-type words are denoted as focus words in the question.

\subsubsection{TF-IDF weighting}

TF-IDF scores for weighting terms are precomputed for all words in all the tables. We do this by treating every table as a unique document. At run-time we discount scores by table length as well as length of the QA pair under consideration to avoid disproportionately assigning high scores to large tables or long MCQs.

\subsubsection{Salience}

The salience of a string for a particular QA pair is an estimate of how relevant it is to the hypothesis formed from that QA pair. It is computed by taking words in the question, pairing them with words in an answer choice and then computing PMI statistics between these pairs from a large corpus. A high salience score indicates words that are particularly relevant for a given QA pair hypothesis.

\subsubsection{Entailment}

To calculate the entailment score between two strings, we use several features, such as overlap, paraphrase probability, lexical entailment likelihood, and ontological relatedness, computed with $\mathrm{n}$-grams of varying lengths.

\subsubsection{Normalization}

All the features in Table 4 produce numerical scores, but the range of these scores vary to some extent. To make our final model more robust, we normalize all feature scores to have a range between 0.0 and 1.0. We do this by finding the maximum and minimum values for any given feature on a training set. Subsequently, instead of using the raw feature value of a feature $f_{d}$, we instead replace it with $\left(f_{d}-\min f_{d}\right) /\left(\max f_{d}-\min f_{d}\right)$.

\section{Experimental Results}

We train FRETS (Section 5) on the TabMCQ dataset (Section 4) using adaptive gradient descent with an L2 penalty of 1.0 and a mini-batch size of 500 training instances. We train two variants: one consisting of all the features from Table 4, the other - a compact model - consisting of the most important features (above a threshold) from the first model by feature-weight. These features are noted by $\boldsymbol{Q}$ in the final column of Table 4 .

We run experiments on three 4th grade science exam MCQ datasets: the publicly available Regents dataset, the larger but unreleased dataset called Monarch, and a third even larger public dataset of Elementary School Science Questions (ESSQ) ${ }^{9}$. For the first two datasets we use the test splits only, since the training sets were directly studied to construct the Aristo Tablestore, which was in turn used to generate our TabMCQ training data. On ESSQ we use all the questions since they are independent of the tables. The Regents test set consists of 129 MCQs, the Monarch test set of 250 MCQs, and ESSQ of 855 MCQs.

Since we are investigating semi-structured models, we compare against two baselines. The first is an unstructured information retrieval method, which uses the Lucene search engine. To apply Lucene to the tables, we ignore their structure and simply use rows as plain-text sentences. The score for top retrieved hits are used to rank the different choices of MCQs. The second baseline is the highly-structured Markov-logic Network (MLN) model from Khot et al. (2015) as reported in Clark et al. (2016), who use the model as a baseline ${ }^{10}$. Note that Clark et al. (2016) achieve a score of 71.3 on Regents Test, which is higher than FRETS' scores (see Table 5), but their results are not comparable to ours because they use an ensemble of algorithms. In contrast, we use a single algorithm with a much smaller collection of knowledge. FRETS rivals the best individual algorithm from their work.

We primarily use the tables from the Aristo Tablestore as knowledge base data in three different settings: with only tables constructed for Regents (40 tables), with only supplementary tables constructed for Monarch (25 tables), and with all ta-

\footnotetext{
${ }^{9}$ http: / / aristo-public-data.s3. amazonaws.com/AI2-Elementary-NDMC-Feb2016. zip

${ }^{10}$ We do not re-implement the MLN, and therefore only cite results from previous work on part of our test suite.
} 


\begin{tabular}{llccc}
\hline Model & Data & Regents Test & Monarch Test & ESSQ \\
\hline \multirow{4}{*}{ Lucene } & Regents Tables & 37.5 & 32.6 & 36.9 \\
& Monarch Tables & 28.4 & 27.3 & 27.7 \\
& Regents+Monarch Tables & 34.8 & 35.3 & 37.3 \\
& Waterloo Corpus & 55.4 & 51.8 & 54.4 \\
\hline MLN & & 47.5 & - & - \\
(Khot et al., 2015) & & & & - \\
\hline \multirow{2}{*}{ FRETS } & Regents Tables & $\mathbf{6 0 . 7}$ & 47.2 & 51.0 \\
(Compact) & Monarch Tables & 56.0 & 45.6 & 48.4 \\
\hline \multirow{2}{*}{ FRETS } & Regents+Monarch Tables & 59.9 & 47.6 & 50.7 \\
& Regents Tables & 59.1 & $\mathbf{5 2 . 8}$ & 54.4 \\
& Monarch Tables & 52.9 & 49.8 & 49.5 \\
& Regents+Monarch Tables & 59.1 & 52.4 & $\mathbf{5 4 . 9}$ \\
\hline
\end{tabular}

Table 5: Evaluation results on three benchmark datasets using different sets of tables as knowledge bases. Best results on a dataset are highlighted in bold.

bles together (all 65 tables; see Section 3). For the Lucene baseline we also experiment with several orders of magnitude more data by indexing over the $5 \times 10^{10}$ words Waterloo corpus compiled by Charles Clarke at the University of Waterloo. Data is not a variable for MLN, since we directly cite results from Clark et al. (2016).

The word vectors we used in soft matching feature variants (i.e., $\diamond$ features from Table 4 ) for all our experiments were trained on 300 million words of Newswire English from the monolingual section of the WMT-2011 shared task data. These vectors were improved post-training by retrofitting (Faruqui et al., 2014) them to PPDB (Ganitkevitch et al., 2013).

The results of these experiments is presented in Table 5. All numbers are reported in percentage accuracy. We perform statistical significance testing on these results using Fisher's exact test with a $\mathrm{p}$-value of 0.05 and report them in our discussions.

First, FRETS - in both full and compact form - consistently outperforms the baselines, often by large margins. For Lucene, the improvements over all but the Waterloo corpus baseline are statistically significant. Thus FRETS is able to capitalize on data more effectively and rival an unstructured model with access to orders of magnitude more data. For MLN, the improvements are statistically significant in the case of Regents and $R e$ gents + Monarch tables. FRETS is thus performing better than a highly structured model while making use of a much simpler data formalism.

Our models are able to effectively generalize. With Monarch tables, the Lucene baseline is little better than random $(25 \%)$. But with the same knowledge base data, FRETS is competitive and sometimes scores higher than the best Lucene or MLN models (although this difference is statisti-

\begin{tabular}{lccc}
\hline Model & REG & MON & ESSQ \\
\hline FRETS & 59.1 & $\mathbf{5 2 . 4}$ & $\mathbf{5 4 . 9}$ \\
w/o tab features & 59.1 & 47.6 & 52.8 \\
w/o row features & 49.0 & 40.4 & 44.3 \\
w/o col features & 59.9 & 47.2 & 53.1 \\
w/o cell features & 25.7 & 25.0 & 24.9 \\
w/o $\diamond$ features & $\mathbf{6 2 . 2}$ & 47.5 & 53.3 \\
\hline
\end{tabular}

Table 6: Ablation study on FRETS, removing groups of features based on level of granularity. $\diamond$ refers to the soft matching features from Table 4. Best results on a dataset are highlighted in bold.

cally insignificant). These results indicate that our models are able to effectively capture both content and structure, reasoning approximately (and effectively) when the knowledge base may not even contain the relevant information to answer a question. The Monarch tables themselves seem to add little value, since results for Regents tables by themselves are just as good or better than Regents+Monarch tables. This is not a problem with FRETS, since the same phenomenon is witnessed with the Lucene baseline. It is noteworthy, however, that our models do not suffer from the addition of more tables, showing that our search procedure over table cells is robust.

Finally, dropping some features in the compact model doesn't always hurt performance, in comparison with the full model. This indicates that potentially higher scores are possible by a principled and detailed feature selection process. In these experiments the difference between the two FRETS models on equivalent data is statistically insignificant.

\subsection{Ablation Study}

To evaluate the contribution of different features we perform an ablation study, by individually removing groups of features from the full FRETS 
model, and re-training. Evaluation of these partial models is given in Table 6. In this experiment we use all tables as knowledge base data.

Judging by relative score differential, cell features are by far the most important group, followed by row features. In both cases the drops in score are statistically significant. Intuitively, these results make sense, since row features are crucial in alignment to questions, while cell features capture the most fine-grained properties. It is less clear which among the other three feature groups is dominant, since the differences are not statistically significant. It is possible that cell features replicate information of other feature groups. For example, the cell answer-type entailment feature indirectly captures the same information as the header answer-type match feature (a column feature). Similarly, salience captures weighted statistics that are roughly equivalent to the coarsegrained table features. Interestingly, the success of these fine-grained features would explain our improvements over the Lucene baseline in Table 5, which is incapable of such fine-grained search.

\section{Conclusions}

We have presented tables as knowledge bases for question answering. We explored a connected framework in which tables are first used to guide the creation of MCQ data with alignment information to table elements, then jointly with this data are used in a feature-driven model to answer unseen MCQs. A central research question of this paper was the trade-off between the degree of structure in a knowledge base and its ability to be harvested or reasoned with. On three benchmark evaluation sets our consistently and significantly better scores over an unstructured and a highly-structured baseline strongly suggest that tables can be considered a balanced compromise in this trade-off. We also showed that our model is able to generalize from content to structure, thus reasoning about questions whose answer may not even be contained in the knowledge base.

We are releasing our dataset of more than 9000 MCQs and their alignment information, to the research community. We believe it offers interesting challenges that go beyond the scope of this paper - such as question parsing, or textual entailment and are exciting avenues for future research.

\section{Acknowledgement}

We'd like to thank AI2 for funding this research and the creation of our MCQ dataset. The first and third authors of this paper were also supported in part by the following grants: NSF grant IIS1143703, NSF award IIS-1147810, DARPA grant FA87501220342. Thanks also go to the anonymous reviewers, whose valuable comments helped to improve the quality of the paper.

\section{References}

Bahadir Ismail Aydin, Yavuz Selim Yilmaz, Yaliang Li, Qi Li, Jing Gao, and Murat Demirbas. 2014. Crowdsourcing for multiple-choice question answering. In Twenty-Sixth IAAI Conference.

Michael J Cafarella, Alon Halevy, Daisy Zhe Wang, Eugene Wu, and Yang Zhang. 2008. Webtables: exploring the power of tables on the web. Proceedings of the VLDB Endowment, 1(1):538-549.

Peter Clark, Oren Etzioni, Tushar Khot, Ashish Sabharwal, Oyvind Tafjord, Peter Turney, and Daniel Khashabi. 2016. Combining retrieval, statistics, and inference to answer elementary science questions. Proceedings of the 30th AAAI Conference on Artificial Intelligence, AAAI-2016.

Peter Clark. 2015. Elementary school science and math tests as a driver for ai: Take the aristo challenge. Proceedings of IAAI, 2015.

Oren Etzioni, Michele Banko, Stephen Soderland, and Daniel S Weld. 2008. Open information extraction from the web. Communications of the ACM, 51(12):68-74.

Anthony Fader, Luke Zettlemoyer, and Oren Etzioni. 2014. Open question answering over curated and extracted knowledge bases. In Proceedings of the 20th ACM SIGKDD international conference on Knowledge discovery and data mining, pages 11561165. ACM.

Manaal Faruqui, Jesse Dodge, Sujay K Jauhar, Chris Dyer, Eduard Hovy, and Noah A Smith. 2014. Retrofitting word vectors to semantic lexicons. arXiv preprint arXiv:1411.4166.

Juri Ganitkevitch, Benjamin Van Durme, and Chris Callison-Burch. 2013. PPDB: The paraphrase database. In Proceedings of NAACL-HLT, pages 758-764, Atlanta, Georgia, June. Association for Computational Linguistics.

Heng Ji and Ralph Grishman. 2011. Knowledge base population: Successful approaches and challenges. In Proceedings of the 49th Annual Meeting of the Association for Computational Linguistics: $\mathrm{Hu}$ man Language Technologies-Volume 1, pages 11481158. Association for Computational Linguistics. 
Tushar Khot, Niranjan Balasubramanian, Eric Gribkoff, Ashish Sabharwal, Peter Clark, and Oren Etzioni. 2015. Exploring markov logic networks for question answering. Proceedings of EMNLP, 2015 .

Dekang Lin and Patrick Pantel. 2001. Discovery of inference rules for question-answering. Natural Language Engineering, 7(04):343-360.

Dan Moldovan, Christine Clark, Sanda Harabagiu, and Steve Maiorano. 2003. Cogex: A logic prover for question answering. In Proceedings of the 2003 Conference of the North American Chapter of the Association for Computational Linguistics on $\mathrm{Hu}$ man Language Technology-Volume 1, pages 87-93. Association for Computational Linguistics.

Srini Narayanan and Sanda Harabagiu. 2004. Question answering based on semantic structures. In Proceedings of the 20th international conference on Computational Linguistics, page 693. Association for Computational Linguistics.

Arvind Neelakantan, Quoc V Le, and Ilya Sutskever. 2015. Neural programmer: Inducing latent programs with gradient descent. arXiv preprint arXiv:1511.04834.

Panupong Pasupat and Percy Liang. 2015. Compositional semantic parsing on semi-structured tables. arXiv preprint arXiv: 1508.00305 .

Rakesh Pimplikar and Sunita Sarawagi. 2012. Answering table queries on the web using column keywords. Proceedings of the VLDB Endowment, 5(10):908-919.

Dan Shen and Mirella Lapata. 2007. Using semantic roles to improve question answering. In EMNLPCoNLL, pages 12-21.

Rohini Srihari and Wei Li. 1999. Information extraction supported question answering. Technical report, DTIC Document.

Huan Sun, Xiaodong He, Wen-tau Yih, Yu Su, and Xifeng Yan. 2016. Table cell search for question answering. In Proceedings of the 25th International Conference on World Wide Web (to appear).

Zareen Syed, Tim Finin, Varish Mulwad, and Anupam Joshi. 2010. Exploiting a web of semantic data for interpreting tables. In Proceedings of the Second Web Science Conference.

Stefanie Tellex, Boris Katz, Jimmy Lin, Aaron Fernandes, and Gregory Marton. 2003. Quantitative evaluation of passage retrieval algorithms for question answering. In Proceedings of the 26th annual international ACM SIGIR conference on Research and development in informaion retrieval, pages 41-47. ACM.
Petros Venetis, Alon Halevy, Jayant Madhavan, Marius Paşca, Warren Shen, Fei Wu, Gengxin Miao, and Chung Wu. 2011. Recovering semantics of tables on the web. Proc. VLDB Endow., 4(9):528-538, June.

Jason Weston, Antoine Bordes, Sumit Chopra, and Tomas Mikolov. 2015. Towards ai-complete question answering: a set of prerequisite toy tasks. arXiv preprint arXiv:1502.05698.

Pengcheng Yin, Nan Duan, Ben Kao, Junwei Bao, and Ming Zhou. 2015a. Answering questions with complex semantic constraints on open knowledge bases. In Proceedings of the 24th ACM International on Conference on Information and Knowledge Management, pages 1301-1310. ACM.

Pengcheng Yin, Zhengdong Lu, Hang Li, and Ben Kao. 2015b. Neural enquirer: Learning to query tables. arXiv preprint arXiv:1512.00965. 